PROCEEDINGS OF THE

AMERICAN MATHEMATICAL SOCIETY

Volume 126, Number 9, September 1998, Pages 2725-2732

S 0002-9939(98)04345-7

\title{
ON THE RELATIVE COMMUTANTS OF SUBFACTORS
}

\author{
M. KHOSHKAM AND B. MASHOOD
}

(Communicated by Palle E. T. Jorgensen)

\begin{abstract}
Let $A \subset B$ be factors generated by a periodic tower $A_{n} \subset B_{n}$ of finite dimensional $C^{*}$-algebras. We prove that for sufficiently large $n, A^{\prime} \cap B$ is *-isomorphic to a subalgebra of $A_{n}^{\prime} \cap B_{n}$.
\end{abstract}

\section{INTRODUCTION}

This paper is mainly concerned with the structure of the relative commutants $A^{\prime} \cap B$ of a pair $A \subset B$ of type $\mathrm{II}_{1}$ factors, generated by an infinite ladder $A_{n} \subset B_{n}$ of finite dimensional $C^{*}$-algebras. If $A_{n} \subset B_{n}$ is obtained by iteration of the basic construction on $B_{0} \subset B_{1}$, then it is a well known result of A. Ocneanu that $A^{\prime} \cap B \subset A_{n}^{\prime} \cap B_{n}$; however, this is not true in general. For example, the tower of higher relative commutants associated with a pair of non-amenable subfactors does not satisfy this property (cf. [8]). We prove a weaker version of this property for general periodic towers. The periodicity is needed to ensure that the Jones index $[A: B]$ is finite. But we do not assume $A_{n} \subset B_{n}$ to be a tower of commuting squares. Specifically, we prove that $A^{\prime} \cap B$ is *-isomorphic to a subalgebra of $A_{n}^{\prime} \cap B_{n}$ for sufficiently large $n$. This will have several interesting consequences, one being a generalization of (Theorem 1.7, [9]). Our main tool is the perturbation technique developed by E. Christensen in [1]. Given $\epsilon>0$, we prove that for sufficiently large $n, A^{\prime} \cap B \stackrel{\epsilon}{\subset} A_{n}^{\prime} \cap B_{n}$; then we are in a situation to use perturbation theory and get the desired isomorphism. The last section of the paper deals with towers of commuting squares. In particular, we obtain a sufficient condition for a tower $A_{n} \subset B_{n}$ to be a tower of commuting squares.

\section{$\S 1$. Notations AND PRELIMINARIES}

Let $B$ be a type $\mathrm{II}_{1}$ factor and let $t r$ be the faithful, normal and normalized trace on $B$. Denote by $L^{2}(B, t r)$ the Hilbert space closure of $B$ under the norm given by the inner product $\langle x, y\rangle=\operatorname{tr}\left(y^{*} x\right)$. Then, $B$ acts on $L^{2}(B, t r)$ by left multiplication, and the identity of $B$ is a cyclic and separating vector for $B$ denoted by $\xi_{0}$. The involution $x \rightarrow x^{*}$ extends to a conjugate linear isometry on $L^{2}(B, t r)$ denoted by $J_{B}$. If $A$ is a von Neumann subalgebra of $B$, let $E_{A}$ be the conditional expectation

Received by the editors February 11, 1997.

1991 Mathematics Subject Classification. Primary 46L37.

Key words and phrases. Subfactors, von Neumann algebras, Jones index, commutant, commuting squares. 
from $B$ onto $A$ associated with the trace, so that $\operatorname{tr}\left(E_{A}(x)\right)=\operatorname{tr}(x)$ for every $x \in B$. The extension of $E_{A}$ to $L^{2}(B, t r)$, denoted by $e_{A}$, is the orthogonal projection of $L^{2}(B, t r)$ onto the closure of $A$ regarded as a subspace of $L^{2}(B, t r)$. Then, $\left\langle B, e_{A}\right\rangle$ the von Neumann algebra generated by $B$ and $e_{A}$ is called the basic construction. The following facts from [3] are frequently used in this the paper and are listed below for convenience.

(a) If $x \in B$, then $x \in A \Leftrightarrow x e_{B}=e_{B} x$,

(b) if $x \in B$, then $e_{A} x e_{A}=E_{N}(x) e_{A}$,

(c) $J_{B}\left\langle B, e_{A}\right\rangle J_{B}=A^{\prime}$

(d) the map $x \rightarrow x e_{A}$ is an isomorphism of $\mathrm{A}$ onto $A e_{A}$,

(e) $e_{A}\left\langle B, e_{A}\right\rangle e_{A}=A e_{A}$

The index of $A$ in $B$, denoted by [B:A], is defined to be $\left(\operatorname{tr}_{A^{\prime}}\left(e_{A}\right)\right)^{-1}$ if $A^{\prime}$ is finite and to be infinite otherwise (cf. [3]). It is a remarkable result of Jones that $[B: A] \in\left\{4 \cos ^{2} \frac{\pi}{n}: n=3,4, \cdots\right\} \cup[4, \infty), A^{\prime} \cap B=\mathbf{C}$ if $[B: A]<4$, and $A^{\prime} \cap B$ is finite dimensional if $[B: A]<\infty$.

If $A \subset B$ are finite dimensional $C^{*}$-algebras with $\left\{e_{i}: i=1,2, \cdots, k\right\}$ and $\left\{f_{j}: j=1, \cdots, l\right\}$ the sets of minimal central projections of $A$ and $B$ respectively, then the inclusion matrix $T_{A}^{B}=\left(a_{i j}\right)$ is defined by

$$
a_{i j}= \begin{cases}0, & \text { if } e_{i} f_{j}=0 \\ \frac{\operatorname{dim} B_{e_{i} f_{j}}}{\operatorname{dim} A_{e_{i} f_{j}}} & \text { if } e_{i} f_{j} \neq 0 .\end{cases}
$$

The matrix $T_{A}^{B}$ is unique up to permutations of the minimal central projections. It is an important fact that if $\left\langle B, e_{A}\right\rangle$ is the basic construction, then $\left\langle B, e_{A}\right\rangle$ is also finite dimensional and $T_{B}^{\left\langle B, e_{A}\right\rangle}=\left(T_{A}^{B}\right)^{t}$. The inclusion $A \subset B$ may also be described by a bipartite graph called the Bratteli diagram of the pair $A \subset B$, with the blocks of $A$ and $B$ forming the vertices of the graph and the edges being the multiplicities of subfactors of $A$ in the subfactors of $B$ (cf.[2],[3]). Increasing sequences of finite dimensional $C^{*}$-algebras $\left(A_{n}\right),\left(B_{n}\right)$, with $A_{n} \subset B_{n}$, are said to constitute a periodic tower of algebras if for some $h$ and all $n$ we have:

$$
T_{B_{n+h}}^{B_{n+h+1}}=T_{B_{n}}^{B_{n+1}}, T_{A_{n}+h}^{A_{n+1+h}}=T_{A_{n}}^{A_{n+1}}, T_{A_{n}}^{B_{n}}=T_{A_{n+h}}^{B_{n+h}} .
$$

If there exists a faithful trace on $\bigcup B_{n}$, then $B=\overline{\bigcup B_{n}}$ and $A=\overline{\bigcup A_{n}}$ are the closures with respect to the weak topology induced by the trace. We say that $A \subset B$ is generated by the tower $A_{n} \subset B_{n}$. If the inclusion matrices are indecomposable and the tower is periodic, then $A$ and $B$ are factors and $[A: B]<\infty$. A diagram

$$
\begin{array}{lll}
A & \subset & B \\
\cup & & \cup \\
C & \subset & D
\end{array}
$$

of finite dimensional $C^{*}$-algebras is called a commuting square with respect to a trace, $t r$, on $B$ if

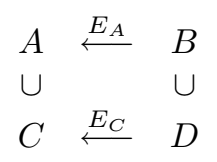

is a commutative diagram, i.e., for each $x \in D, E_{A}(x) \in C$. The expectations $E_{A}$ and $E_{C}$ correspond to the trace, and we assume $t r$ is a Markov trace. 


\section{§2. MAIn RESULtS}

Near inclusions $A \stackrel{\delta}{\subset} B$ of pairs of von Neumann subalgebras of a type $\mathrm{II}_{1}$ von Neumann algebra $C$ were studied by E. Christensen in [1]. The relation $A \stackrel{\delta}{\subset} B$ means that for every $x \in A_{1}$, the unit ball of $A$, there exists an element $y \in B$ such that $\|x-y\|_{2}^{t r}<\delta$, where $t r$ is the faithful, normal, and normalized trace on $C$. If $E_{B}$ denotes the canonical trace preserving conditional expectation from $C$ onto $B$, then

$$
A \stackrel{\delta}{\subset} B \Longleftrightarrow\left\|x-E_{B}(x)\right\|_{2}^{t r}<\delta \forall x \in A_{1} .
$$

As we are considering pairs of factors generated by finite dimensional algebras, we only need to consider inclusions $A \stackrel{\delta}{\subset} B$ with $B$ finite dimensional. We need to modify several perturbations lemmas from [1]. However, the main ideas remain the same. Given $\delta>0$, the following function is frequently needed:

$$
\gamma(\delta)=2^{\frac{1}{4}} \delta^{\frac{1}{2}}\left(1-2^{\frac{1}{4}} \delta^{\frac{1}{2}}\right)^{-1} .
$$

Lemma 2.1. Let $B$ be a finite dimensional subalgebra of a type $\mathrm{II}_{1}$ von Neumann algebra $C$. If tr is the faithful, normal, and normalized trace on $B$, then there exists a semifinite trace, $t^{\prime}$, on $\left\langle C, e_{B}\right\rangle$ such that

$$
\begin{aligned}
\operatorname{tr}^{\prime}\left(x e_{B}\right) & =\operatorname{tr}(x) \forall x \in C, \\
\operatorname{tr}^{\prime}\left(e_{B}\right) & =1 .
\end{aligned}
$$

Proof. Let $e_{1}, e_{2}, \cdots, e_{m}$ be the minimal central projections of $B$. By [3, Proposition 3.1.5], $f_{k}=J_{C} e_{i} J_{C}, 1 \leq k \leq m$, are the minimal central projections of the semifinite von Neumann algebra $\left\langle C, e_{B}\right\rangle$. Hence, there exists a unique faithful normal semifinite trace, $t r^{\prime}$, on $\left\langle C, e_{B}\right\rangle$ such that $\operatorname{tr}^{\prime}\left(f_{k} e_{B}\right)=\operatorname{tr}\left(e_{k}\right)$. Using the *-isomorphism $x f_{k} e_{B} \rightarrow E_{B}(x) e_{k}$ from $\left\langle C, e_{B}\right\rangle f_{k} e_{B}$ onto $B e_{k}$ (cf. [3]) and the uniqueness of the trace on the factor $B e_{k}$, we have that $\operatorname{tr}^{\prime}\left(x f_{k} e_{B}\right)=\operatorname{tr}\left(E_{B}(x) e_{k}\right)$ for all $x \in C$. Given $x \in C$, we have

$$
\begin{aligned}
\operatorname{tr}^{\prime}\left(x e_{B}\right) & =\operatorname{tr}^{\prime}\left(\sum_{k=1}^{m} x f_{k} e_{B}\right) \\
& =\sum_{k=1}^{m} \operatorname{tr}^{\prime}\left(x f_{k} e_{B}\right) \\
& =\sum_{k=1}^{m} \operatorname{tr}\left(E_{B}(x) e_{k}\right) \\
& =\operatorname{tr}\left(E_{B}(x) \sum_{k=1}^{m} e_{k}\right)=\operatorname{tr}(x) .
\end{aligned}
$$

Let $x=1$ in the above to get $\operatorname{tr}^{\prime}\left(e_{B}\right)=1$.

Lemma 2.2. Let $B, C$, and $e_{1}, e_{2}, \cdots, e_{m}$ be as above. Suppose that $A$ is a von Neumann subalgebra of $C$ such that $A \stackrel{\delta}{\complement} B$ with $\delta<\frac{1}{\sqrt{2}}$ and such that for every minimal projection $q \in B, \gamma^{2}(\delta)<\operatorname{tr}(q)$. Then, there exists a $*$-homomorphism $\varphi$ from $A$ into $B$. Moreover, for each $x$ in the unit ball of $A$ we have:

$$
\|\varphi(x)-x\|<26 \gamma(\delta)+\delta, \quad \varphi(I)=I .
$$


Proof. If $u \in A$ is unitary, then

$$
\operatorname{tr}\left(I-E_{B}\left(u^{*}\right) E_{B}(u)\right)=\left\|u-E_{B}(u)\right\|_{2}^{t r}<\delta^{2} .
$$

If $t r^{\prime}$ is as in Lemma 2.1, then we have

$$
\begin{aligned}
\operatorname{tr}^{\prime}\left(\left(e_{B}-u^{*} e_{B} u\right)^{2}\right) & =\operatorname{tr}^{\prime}\left(e_{B}+u^{*} e_{B} u-u^{*} e_{B} u e_{B}-e_{B} u^{*} e_{B} u\right) \\
& =2 \operatorname{tr}\left(e_{B}\left(1-E_{B}\left(u^{*}\right) E_{B}(u)\right)\right. \\
& =2 \operatorname{tr}\left(I-E_{B}\left(u^{*}\right) E_{B}(u)\right) \\
& <2 \delta^{2} .
\end{aligned}
$$

As in [1, Theorem 3.1], we can find an element $k$ of minimal trace norm \|\|$_{2}^{t r^{\prime}}$ in the ultrastrong closed convex hull of the set $\left\{u^{*} e_{B} u: u\right.$ unitary in $\left.B\right\}$ such that $k \in A^{\prime} \cap\left\langle A, e_{B}\right\rangle$ and

$$
0 \leq k \leq I, \quad\left\|k-e_{B}\right\|_{2}^{t r^{\prime}}<2^{\frac{1}{2}} \delta .
$$

Since $\operatorname{tr}^{\prime}\left(e_{B}\right)=1$, by the discussion preceding [1, Lemma 4.1] there exists a projection $q \in A^{\prime} \cap\left\langle A, e_{B}\right\rangle$ such that

$$
\left\|q-e_{B}\right\|_{2}^{t r^{\prime}} \leq \gamma(\delta) \quad \text { and } \quad\left|1-t r^{\prime}(q)\right|<\gamma(\delta)^{2} .
$$

Let $\left\{f_{1}, \ldots, f_{m}\right\}$ be the set of minimal central projection of $\left\langle C, e_{B}\right\rangle$ (see Lemma 2.1). Then,

$$
\begin{aligned}
\left|\operatorname{tr}^{\prime}\left(q f_{n}\right)-\operatorname{tr}^{\prime}\left(e_{B} f_{n}\right)\right| & \left.=\mid \operatorname{tr}^{\prime}\left(\left(q-e_{B}\right) f_{n}\right)\right) \mid \\
& \leq\left|\operatorname{tr}^{\prime}\left(q-e_{B}\right)\right| \\
& =\left|1-\operatorname{tr}^{\prime}(q)\right| \\
& <\gamma(\delta)^{2} .
\end{aligned}
$$

Since the factor $\left\langle C, e_{B}\right\rangle f_{k}$ is isomorphic to $B e_{k}$ and $\operatorname{tr}(p)>\gamma(\delta)^{2}$ for every projection $p \in B$, the above inequality implies that $\operatorname{tr}^{\prime}\left(q f_{k}\right)=t r^{\prime}\left(e_{B} f_{k}\right)$. Hence, $q f_{k} \sim e_{B} f_{k}$. Let $v_{k} \in\left\langle C, e_{B}\right\rangle f_{k}$ be a partial isometry such that $v_{k} v_{k}^{*}=q f_{k}$ and $v_{k}^{*} v_{k}=e_{B} f_{k}$. Then, $q \sim e_{B}$ via the partial isometry $v=v_{1}+v_{2}+\cdots+v_{m}$. It is easy to check that $m \rightarrow v^{*} m v$ is a homomorphism from $A$ into $\left\langle C, e_{B}\right\rangle e_{B}=B e_{B}$. Let $\varphi$ be the composition of this with the canonical identification of $B e_{B}$ with $B$. If $x$ is in the unit ball of $A$, then by [2, Lemma 4.1], $\left\|\varphi(x)-E_{A}(x)\right\|<26 \gamma(\delta)$. Since $\left\|x-E_{A}(x)\right\|<\delta$, the inequality (4) follows.

Proposition 2.3. Let $A \subset B$ be factors generated by the tower $A_{n} \subset B_{n}$ such that $[B: A]<\infty$. Then, given $\epsilon>0$, there exists $N>0$ such that:

$$
A^{\prime} \cap B \stackrel{\epsilon}{\subset} A_{n}^{\prime} \cap B_{n} \forall n>N .
$$

Proof. Since $B=\overline{\bigcup B_{n}}$, given $x$ in the unit ball of $A^{\prime} \cap B$ there exist an integer $n$ and $y \in B_{n}$ such that $\|x-y\|_{2}^{t r}<\frac{\epsilon}{3}$. Hence, $\left\|x-E_{B_{n}}(x)\right\|_{2}^{t r}<\frac{2 \epsilon}{3}$. Since $[B: A]<\infty$ by [3, Corollary 2.2.3] $A^{\prime} \cap B$ is finite dimensional, and we may use a standard compactness argument and find a positive $N$ such that

$$
\left\|x-E_{B_{n}}(x)\right\|_{2}^{t r}<\epsilon \forall x \in\left(A^{\prime} \cap B\right)_{1} \quad \text { and } \forall n>N .
$$

It is easy to see that if $x \in A^{\prime} \cap B$, then $E_{B_{n}}(x) \in A_{n}^{\prime} \cap B_{n}$ and hence $A^{\prime} \cap B \subseteq A_{n}^{\prime} \cap B_{n}$ by (6) and (1). 
Lemma 2.4. Let $A \subset B$ be factors generated by $A_{n} \subset B_{n}$. If $\lim \sup \left\|T_{A_{n}}^{B_{n}}\right\| \leq \infty$, then $[A: B]<\infty$.

Proof. It follows from [5, Propositions 2.6 and 3.4] that the entropy $H(B, A) \leq$ $\limsup \left\|T_{A_{n}}^{B_{n}}\right\|$. If $A^{\prime} \cap B$ had a completely non-atomic part, then as in the proof of $[5$, Theorem 4.4] we conclude that $H(B, A)=\infty$, which contradicts the earlier statement. Hence, $A^{\prime} \cap B$ must be atomic and by [5, Theorem 4.4], we conclude that $[B: A]<\infty$.

Corollary 2.5. Let $A \subset B$ be generated by a periodic tower $A_{n} \subset B_{n}$. Then, given $\varepsilon>0$, there exists $N$ such that

$$
A^{\prime} \cap B \stackrel{\varepsilon}{\complement} A_{n}^{\prime} \cap B_{n} \forall n>N .
$$

Proof. If $A_{n} \subset B_{n}$ is periodic, then the hypothesis of Lemma 2.4 is satisfied and hence $[A: B]<\infty$. Now Proposition 2.3 can be used.

Theorem 2.6. Let $A \subset B$ be factors generated by $A_{n} \subset B_{n}$ and let $\left\{e_{n}^{k}\right\}_{k}$ be the set of minimal central projections of $A_{n}$. Suppose that

(a) $[B: A]<\infty$,

(b) $\lim _{k, n} \inf \left(\operatorname{tr}\left(e_{n}^{k}\right)\right)>0$,

(c) there exists $r>0$ such that for every $n$ and every minimal projection $p \in$ $A_{n}^{\prime} \cap B_{n}$ we have $\operatorname{tr}(p)>r$.

Then $A^{\prime} \cap B$ is $*$-isomorphic to a subalgebra of $\left(A_{n}^{\prime} \cap B_{n}\right)_{e_{n}^{k}}$ for sufficiently large $n$.

Proof. Choose $d>0$ such that $\lim _{k, n} \inf \operatorname{tr}\left(e_{n}^{k}\right)>d>0$ and choose $\epsilon>0$ such that $\epsilon<\frac{1}{\sqrt{2}}, \gamma^{2}(\epsilon)<r$, and $\gamma(\epsilon)+\epsilon<\operatorname{tr}(p)$ for every minimal projection $p$ in the finite dimensional algebra $A^{\prime} \cap B$. Next choose $\delta>0$ such that $\frac{\delta}{d}<\epsilon$. By Proposition 2.3 there exists $N$ such that $A^{\prime} \cap B \stackrel{\delta}{\complement} A_{n}^{\prime} \cap B_{n}$ when $n>N$. Let $\tilde{\operatorname{tr}} r(x)=\frac{\operatorname{tr}(x)}{\operatorname{tr}\left(e_{n}^{k}\right)}$ for $x \in\left(B_{n}\right) e_{n}^{k}$. Then $\tilde{t} r_{r}$ is the canonical normalized trace on $\left(B_{n}\right)_{e_{n}^{k}}$, and we obtain

$$
\left(A^{\prime} \cap B\right)_{e_{n}^{k}} \frac{\delta}{d}\left(A_{n}^{\prime} \cap B_{n}\right)_{e_{n}^{k}} \forall k
$$

where the above near inclusion is with respect to the $\tilde{t} r$ norm. Since, $\frac{\delta}{d}<\epsilon$ we have that

$$
\left(A^{\prime} \cap B\right)_{e_{n}^{k}} \stackrel{\epsilon}{\complement}\left(A_{n}^{\prime} \cap B_{n}\right)_{e_{n}^{k}} \forall k, n>N .
$$

By the choice of $\epsilon$ the hypothesis of Lemma 2.2 is satisfied, and hence there exists a $*$-homomorphism $\varphi$ from $\left(A^{\prime} \cap B\right)_{e_{n}^{k}}$ into $\left(A_{n}^{\prime} \cap B_{n}\right)_{e_{n}^{k}}$. If $\varphi(x)=0$ for some $x \in\left(A^{\prime} \cap B\right)_{e_{k, n}}$, then $\varphi(p)=0$ for some projection. By $(4), \operatorname{tr}(p)<\gamma(\epsilon)+\epsilon$. This contradicts the choice of $\epsilon$. Hence, $\varphi$ must be one to one. Finally, as $e_{n}^{k} \in A$ and $A$ is a factor, $\left(A^{\prime} \cap B\right)_{e_{n}^{k}}$ is $*$-isomorphic to $A^{\prime} \cap B$, and the proof is complete.

Theorem 2.7. Suppose that $A \subset B$ is generated by a periodic tower $A_{n} \subset B_{n}$ of finite dimensional $C^{*}$-algebras, then $A^{\prime} \cap B$ is $*$-isomorphic to a subalgebra of $A_{n}^{\prime} \cap B_{n}$.

Proof. Since $A_{n} \subset B_{n}$ is periodic by Corollary 2.5, the index $[B: A]$ is finite. Conditions (b) and (c) of Theorem 2.6 are satisfied here because the inclusion matrices are periodic. We refer to [9] for a proof of this fact. Hence the result follows from Theorem 2.6. 
The following generalizes Theorem 1.7 of [9] in the sense that $A_{n} \subset B_{n}$ is not assumed to be a tower of commuting squares.

Corollary 2.8. If $A \subset B$ and $A_{n} \subset B_{n}$ are as above and there exists $m>0$ such that $\left(A_{n}^{\prime} \cap B_{n}\right)_{e_{k, n}}=\mathbf{C}$ for some $k$ and every $n>m$. Then $A^{\prime} \cap B=\mathbf{C}$.

Proof. Follows directly from Theorems 2.5 and 2.7.

Corollary 2.9. If $A \subset B$ and the tower $A_{n} \subset B_{n}$ are as before, and for a sequence $\left(n_{k}\right)$ the inclusion matrix $T_{A_{n_{k}}}^{B_{n_{k}}}$ has a column (or a row) of exactly one nonzero entry equal to one, then $A^{\prime} \cap B=\mathbf{C}$.

Proof. Our hypothesis implies that for some $l,\left(A_{n_{k}}^{\prime} \cap B_{n_{k}}\right)_{e_{l, n_{k}}}=\mathbf{C}$ for all $n_{k}$. Now the result follows from Corollary 2.8.

Proposition 2.10. Let $A \subset B$, with $[B: A]<\infty$, be factors generated by a tower $A_{n} \subset B_{n}$ of commuting squares. Let $\left\{e_{n}^{k}\right\}$ and $\left\{f_{n}^{l}\right\}$ be the set of minimal central projections of $A_{n}$ and $B_{n}$ respectively and suppose that $\lim _{k, n} \inf \left(\operatorname{tr}\left(e_{n}^{k}\right)\right)>0$. Then, for sufficiently large $n, A^{\prime} \cap B$ is *-isomorphic to a subalgebra of $A_{n}^{\prime} \cap B_{n}$.

Proof. By [5, Proposition 2.6] $[B: A]=\lim \lambda\left(B_{n}: A_{n}\right)^{-1}$, and by $[6$, Theorem 2.3] $\lambda\left(B_{n}, A_{n}\right)^{-1}=\max _{l}\left(\sum_{k} a_{k l}^{2} \operatorname{tr}\left(e_{n}^{k}\right) / \operatorname{tr}\left(e_{n}^{k} f_{n}^{l}\right)\right)$ where $a_{k l}^{2}$ is just the dimension of $\left(A_{n}^{\prime} \cap B_{n}\right) e_{n}^{k} f_{n}^{l}$. Hence, there exists $N$ such that for every $n>N$ we have

$$
a_{k l}^{2} \operatorname{tr}\left(e_{n}^{k}\right) / \operatorname{tr}\left(e_{n}^{k} f_{n}^{l}\right) \leq[B: A] .
$$

Equivalently, for $n>N$

$$
\frac{\operatorname{tr}\left(e_{n}^{k} f_{n}^{l}\right)}{a_{k l}^{2}} \geq \frac{\operatorname{tr}\left(e_{n}^{k}\right)}{[B: A]} .
$$

Now the left hand side of the above inequality is the trace of minimal projections in $\left(A_{n}^{\prime} \cap B_{n}\right) e_{n}^{k} f_{n}^{l}$. Given that $\liminf \operatorname{tr}\left(e_{n}^{k}\right)>0$, it follows that condition (c) of Theorem 2.6 is satisfied here, and the result follows from that theorem.

\section{§3. REMARKS ON COMMUTING SQUARES}

An important tool in construction and the study of subfactors is the concept of commuting squares (see Section 1 for the definition), developed my M. Pisner and S. Popa (cf. [5]), and used by many authors in relation with Jones index theory (cf. [4], [7], [9]). The construction and classification of subfactors are closely related to those of commuting squares. The results of this section are along these lines. If $B_{2}=\left\langle B_{1}, e_{1}\right\rangle$ is the basic construction on $A_{1} \subset B_{1}$ and if $A_{2}=\left\langle A_{1}, e_{1}\right\rangle$, then the following is a commuting square:

$$
\begin{array}{ccc}
A_{2} & \subset & B_{2} \\
\cup & & \cup \\
A_{1} & \subset & B_{1}
\end{array}
$$

By iterating the above procedure we obtain the tower $A_{n} \subset B_{n}$ of commuting squares. Note that the algebra $A_{n}$ contains the set $\left\{e_{1}, e_{2}, \ldots, e_{n-2}\right\}$ of projections corresponding to the basic construction. We want to show that the converse is also true in the following sense. Namely, let the tower $B_{n} \subset B_{n+1}$ be obtained by iterating the basic construction on the pair of finite dimensional $C^{*}$-algebras $B_{1} \subset B_{2}$, and let $\left\{e_{n}\right\}$ be the corresponding Jones' projection. Suppose that $\left\{A_{n}\right\}$ is an increasing sequence of $C^{*}$-algebras with $A_{n} \subset B_{n}$ such that for $n \geq 3, A_{n}$ 
contains the set $\left\{e_{1}, e_{2}, \ldots, e_{n-2}\right\}$. Set $A=\overline{\bigcup A_{n}}, B=\overline{\bigcup B_{n}}$ and $\tilde{A}_{n}=A \cap B_{n}$, where the closures are in the sense of the weak operator topology. With these notations, we state the following.

Proposition 3.1. $\tilde{A}_{n} \subset B_{n}$ is a tower of commuting squares. Moreover, if $[B: A]$ $<\infty$, then the tower $\tilde{A}_{n} \subset B_{n}$ is periodic.

Proof. We must show that if $y \in \tilde{A}_{n}$, then $E_{B_{n-1}}(y) \in \tilde{A}_{n-1}$. Since $e_{n} e_{n-1} e_{n}=\lambda e_{n}$ with $\lambda^{-1}=\left\|T_{B_{1}}^{B_{2}}\right\|$, we have that

$$
\begin{aligned}
e_{n} e_{n-1} y e_{n-1} e_{n} & =e_{n} E_{B_{n-1}}(y) e_{n-1} e_{n} \\
& =E_{B_{n-1}}(y) e_{n} e_{n-1} e_{n} \\
& =\lambda E_{B_{n-1}}(y) e_{n} .
\end{aligned}
$$

Hence, $e_{n} E_{B_{n-1}}(y) \in \tilde{A}_{n+2} \subset A$. It follows that $e_{m} E_{B_{n-1}}(y) \in A$ for all $m \geq$ $n$. Hence, $e_{n} \vee e_{n+1} \vee \cdots \vee e_{m} E_{B_{n-1}}(y) \in A$ for $m>n$. Since $\operatorname{tr}\left(1-e_{n+1} \vee\right.$ $\cdots \vee e_{m}$ ) converges to zero as $m$ tends to infinity (cf. $\S 4$, [2]), the projections $e_{n} \vee \cdots \vee e_{m}$ converge to the identity as $m$ tends to infinity. Hence, $E_{B_{n-1}}(y) \in$ $A \cap B_{n-1}=\widetilde{A}_{n-1}$, proving the first statement. When $[B: A]<\infty$ the periodicity is a consequence of the fact that $[B: A]=\lim \left[B_{n}: A_{n}\right]$ (cf. [5]).

We obtain the following corollary regarding intermediate subfactors.

Corollary 3.2. Let $A_{n} \subset B_{n}$ be obtained by the basic construction and let $C$ be a middle subfactor, $A \subset C \subset B$. Then there exists a periodic tower of commuting squares $A_{n} \subset C_{n} \subset B_{n}$ such that $C=\lim C_{n}$.

Proof. Let $C_{n}=B_{n} \cap C$ and apply the previous lemma.

The following theorem shows that for factors $A \subset B$ generated by a tower $A_{n} \subset$ $B_{n}$ the existence of proper intermediate subfactors may be reduced to a finite dimensional problem.

Theorem 3.3. Let $A \subset B$ be generated by the tower $A_{n} \subset B_{n}$ given by the basic construction. Then there exists a proper middle subfactor $C$ if and only if, for some $n$, there exist $C^{*}$-algebras $C_{n} \subset C_{n+2}$ such that

$$
\begin{aligned}
A_{n+2} & \subset C_{n+2} \\
A_{n} & \subset C_{n} \quad B_{n+2},
\end{aligned}
$$

with $T_{C_{n}}^{B_{n}}=T_{C_{n+2}}^{B_{n+2}}$.

Proof. The only if part follows from the previous corollary. Now suppose that $C_{n} \subset$ $C_{n+2}$ are as in the statement of the theorem. Since the identity is in $A_{n+2} \cap C_{n+2}$, for every minimal central projection $q$ in $C_{n+2}, q e_{n}$ is a nonzero projection. Then $e_{n} E_{B_{n}}\left(C_{n+1}\right)=e_{n} C_{n+1} e_{n}$ is a subset of $e_{n}\left(C_{n+2}\right) e_{n}=\left(C_{n}\right)_{e_{n}}$. The last equality holds because

$$
\left(C_{n}\right)_{e_{n}} \subset\left(C_{n+2}\right)_{e_{n}} \subset\left(B_{n+2}\right)_{e_{n}}=\left(B_{n}\right)_{e_{n}}
$$


and $T_{C_{n}}^{B_{n}}=T_{C_{n+2}}^{B_{n=2}}$. Therefore, $E_{B_{n}}\left(C_{n+1}\right) \subseteq C_{n}$. But $C_{n} \subseteq E_{B_{n}}\left(C_{n+2}\right)$, whence $E_{B_{n}}\left(C_{n+1}\right)=C_{n}$. This shows that

$$
\begin{array}{ccc}
C_{n+1} & \subset & B_{n+1} \\
\cup & & \cup
\end{array}
$$

is a commuting square, and it is easy to see that $C_{n+2}=\left\langle C_{n+1}, e_{n}\right\rangle$. Then $C$ can be constructed inductively.

\section{REFERENCES}

[1] Christensen, E., Subalgebras of a finite algebra., Math. Ann. 243 (1979), 17-29. MR 80i: 46051

[2] Goodman, F., De La Harpe, P.S., Jones, V.F.R., Coxeter graphs and towers of algebras, Math. Sci. Res. Hist. Publ., Springer-Verlag 14 (1989). MR 91c:46082

[3] Jones, V., Index for subfactors, Invent. Math. 72 (1983), 1-25. MR 84d:46097

[4] Ocneanu, A., Quantized groups, string algebras and Galois theory for von-Neumann algebras, Operator algebras and applications, London Math. Soc. Lecture Notes ser. 136 119-172, Cambridge Univ. Press, 1988. MR 91k:46068

[5] Pimsner, M., Popa, S., Entropy and index for subfactors., Ann. Sci. Ecole Norm. Sup. 19 (1986), 57-106. MR 87 m:46120

[6] Pimsner, M., Popa, S., Finite dimensional approximation for pair of algebras and obstruction for index, J. Func. Anal. 98 (1991), 270-291. MR 92g:46076

[7] Popa, S., Classification of subfactors, reduction to commuting squares, Invet. Math. 101 (1990), 19-43. MR 91h:46109

[8] Popa, S., Classification of amenable subfactors of type II, Acta Math. 172 (1994), 163-255. MR 95f: 46105

[9] Wenzl, H., Representation of Hecke algebras and subfactors, Thesis, University of Pennsylvania.

Department of Mathematics, University of Saskatchewan, 106 Wiggins Road, Saskatoon, Saskatchawan, Canada S7N 5E6

E-mail address: khoshkam@math.usask.ca 